\title{
Systematic review for orthodontic and orthopedic treatments for anterior open bite in the mixed dentition
}

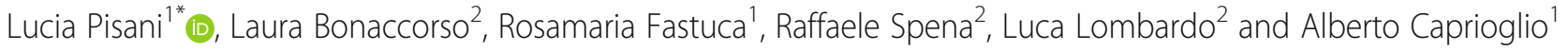

\begin{abstract}
Background: The treatment options for the early treatment of anterior open bite are still controversial. The aim of this study was to evaluate the actual available evidence on treatments of anterior open bite in the mixed dentition in order to assess the effectiveness of the early treatment in reducing open bite, the most efficacious treatment strategy and the stability of the results.

Materials and methods: A literature survey was done on November 15, 2015, by means of appropriate Medical Subject Headings (MeSH) using the following databases: PubMed, EMBASE, Cochrane Library, LILACS, VHL, and WEB OF SCIENCE.

Randomized clinical trials and studies with a control group (treated or untreated) were then selected by two authors. Trials including patients with syndromes or in the permanent dentition and studies concerning treatment with extractions, full-fixed appliances, or surgery were not considered.

Full articles were retrieved for abstracts or titles that met the initial inclusion criteria or lacked sufficient detail for immediate exclusion.
\end{abstract}

Results: Two thousand five hundred sixty-nine studies about open bite were available; the search strategy selected 240 of them.

Twenty-four articles have been judged suitably for the final review, and their relevant data were analyzed.

Discussion: Although this review confirms the effectiveness of early treatment of open bite, particularly when no-compliance strategies are employed, meta-analysis was unfeasible due to lack of standardization, important methodological limitations, and shortcomings of the studies.

Conclusions: A more robust approach to trial design in terms of methodology and error analysis is needed. Besides, more studies with longer periods of follow-up are required.

Keywords: Early treatment, Open bite, Systematic review, Quality analysis

\section{Review}

\section{Background}

Anterior open bite is a malocclusion characterized by a deficiency in the normal vertical overlap between antagonist incisal edges when the posterior teeth are in occlusion [1].

\footnotetext{
* Correspondence: lucia.pisani88@gmail.com

'Division of Orthodontics, Department of Surgical and Morphological

Sciences, University of Insubria, Varese, Italy

Full list of author information is available at the end of the article
}

Dental and dentoalveolar open bite is the result of a mechanical blockage of the vertical development of the incisors and the alveolar component while skeletal relationships are normal; skeletal open bite is determined by a vertical skeletal discrepancy [2]. However, in most cases, the distinction is not clear since malocclusion presents both dental and skeletal components [3].

Skeletal open bite is characterized by increased lower anterior facial height and gonial angle, short mandibular ramus, and increased posterior dentoalveolar height. Concomitant transverse discrepancies may also be present [4]. Additional features are lip incompetence, profile convexity, 
marked incisors labial inclination and crowding $[5,6]$. For these reasons, anterior open bite is a major cause of masticatory and phonatory function impairment and also causes considerable esthetic issues to the affected patients [7].

Etiology involves the interaction of environmental factors such as prolonged sucking habits, mouth breathing, tongue or lip thrusting, and eruption disturbances with a genetically determined vertical facial grow pattern [2, 6, 8-13].

Several authors emphasized that a skeletal open bite should be treated in the mixed dentition in order to take advantage of the active growth producing faster and more stable results and to reduce the burden of treatment in the permanent dentition $[14,15]$. Various approaches have been proposed on this purpose.

Vertical chin cup [16], bite blocks [17-24], chewing exercises [25], and extractions and mesialization of posterior teeth [26] have been advocated to achieve relative and true intrusion of molars. Palatal cribs and spurs are used to prevent persisting sucking habits or tongue thrust in order to promote a normal anterior segment development [27-35]. Functional therapy would be useful in correcting the faulty postural activity of the orofacial musculature and the associated skeletal deformity [36-41].

However, treatment of skeletal anterior open bite is still one of the most difficult challenges for the orthodontist. Effectiveness and long-term stability of available treatment modalities are critical issues because of the lack of a strong scientific evidence [42, 43].

The objective of this work was to perform a systematic review of the literature in order to evaluate the actual available evidence on treatments of anterior open bite in the mixed dentition and to assess the effectiveness of the early treatment in reducing open bite and divergency, the most efficacious treatment strategy and the stability of the results.

\section{Materials and methods}

This systematic review was written according to the PRISMA guidelines [44].

The search strategy was based on the National Health Service Center for Reviews and Dissemination guidelines [45].

A first survey of all articles published up to November 2015 about anterior open bite was performed by using the following databases: PubMed, EMBASE, Cochrane Library, LILACS, VHL, and WEB OF SCIENCE.

The search strategy for PubMed was then improved according to Cochrane Collaboration guidelines using the Medical Subject Headings (MeSH) terms "early treatment" and "dentition, mixed," crossed with combinations of the MeSH term "open bite".

The key words used to identify the corresponding studies in the other databases were: "open bite" and "mixed dentition".
References from original papers and reviews were checked.

Randomized controlled trials (RCTs) and prospective or retrospective studies with a control group (treated or untreated) reporting data on the effects of the treatment in the mixed dentition were included.

Descriptive studies, case reports, case series, debate articles, and studies concerning treatment in the permanent dentition, with extractions, with full-fixed appliances, or surgically assisted were excluded. Studies including patients with cleft lip or palate or both or other syndrome associated with craniofacial anomalies were not considered.

Duplicate reports were excluded.

Two authors (L.P. and L.B.) screened the titles and abstracts and independently assessed the eligibility of all the reports. Full articles were retrieved for abstracts or titles that met the initial inclusion criteria or lacked sufficient details for immediate exclusion.

The articles that were judged suitably for the final review analysis were read, and their relevant data were retrieved for pooling.

Data were collected on study design, treatment modalities, characteristics of the sample, methods of measurements, success rate, decrease of open bite and divergency, treatment duration, side effects and costs, and stability.

A quality evaluation modified by the protocol described by Antczak [46] and Jadad [47] was performed for each article. This considered sample size, selection description, withdrawals, validity of the methods, method error analysis, blinding in measurements, and adequate statistics. The quality was categorized as low, medium, and high.

\section{Results}

As shown in the flow chart (Fig. 1), 2569 articles about anterior open bite were available in the literature.

The combination of MeSh terms resulted in 240 articles.

According to the inclusionary/exclusionary criteria, 25 articles, published between 1983 and 2015, were selected.

One was excluded because two groups were treated with a multibracket appliance.

Twenty four articles were then considered for the final review analysis.

\section{Trial design and treatment modalities}

Data about trial design and treatment modalities are shown in Table 1.

Three randomized controlled trials were found [23, 28, 40].

The effects of Quad Helix with crib (Q-H/C) were examined by three studies $[29,30,35]$. Other trials compared them versus those of removable palatal crib (RPC) [32] and open bite bionator (OBB) [31].

OBB was tested by two further authors alone $[16,41]$ in combination with a high-pull headgear (HPH) [39]. Two 


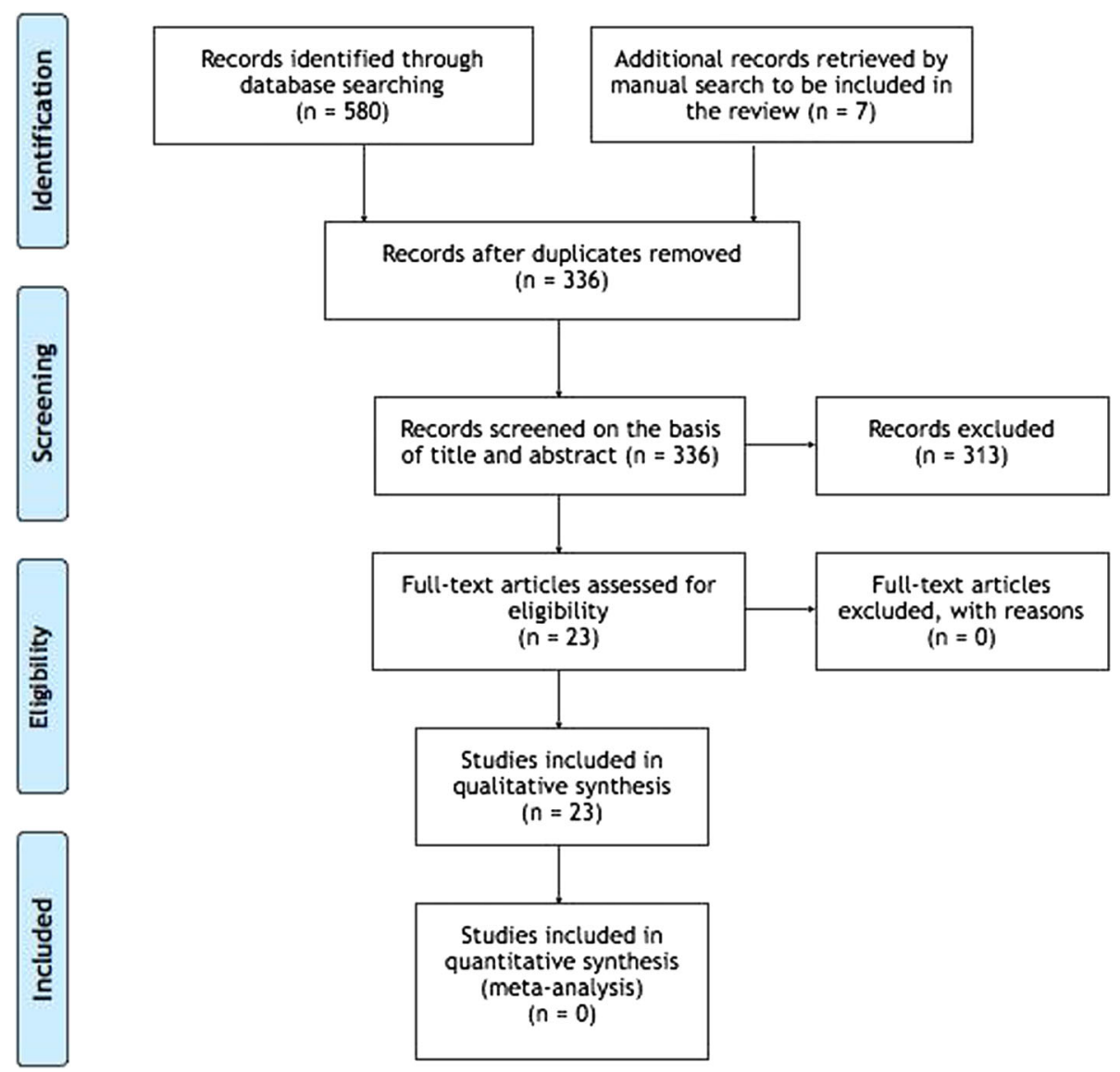

Fig. 1 Flow chart of the selection of the studies

studies described the effects of RPC associated with a vertical chin cup (RPC + VCC) $[27,28]$ and one compared it versus fixed palatal crib and VCC (FPC + VCC) [33].

One trial assessed the effects of bonded spurs in combination with VCC (BS + VCC) [34], one those of Teucher appliance (A-HPH) [38], and one those of VCC alone [16].

The results of Fränkel appliance (FR) was described by three studies [19, 36, 40].

Posterior bite blocks at 5 or $10 \mathrm{~mm}$ in height (PBB5, PBB10) [20] and magnetic bite blocks (MBB) [21] were tested. The effects of MBB were compared versus spring-loaded bite blocks (SLBB) [18, 23], PBB [37], and rapid molar intruder (RMI) [24].

This latter was tested versus a control group in one trial [22] where a further group enrolled older patients treated with RMI and a multibracket appliance. Since the application of a multibracket appliance was not suitable with our inclusion criteria, only data relative to RMI group and control group were considered.

Finally, Ișcan et al. compared the association PBB + VCC versus SLBB [19].

\section{Characteristics of the participants}

Five authors [27, 28, 33, 34, 40] included only the subjects with anterior open bite greater than $1 \mathrm{~mm}$.

Eighteen studies [16-20, 22-24, 29-32, 35-38, 40, 41] had skeletal anterior open bite in their inclusion criteria. For the remaining studies [21, 27, 28, 33, 34, 39], the inclusion criterion was anterior open bite independently of the type.

Bad habits were an exclusion criterion in five studies $[17,20,22,28,37]$ while four trials $[29,30,32,35]$ included only patient with thumb-sucking habit and related constricted maxillary arch before treatment. The remaining studies did not evaluate the presence of bad habits.

Three articles $[22,28,34]$ excluded subjects with maxillary constriction.

Full eruption of the permanent first molars and incisors was an inclusion criterion for the seven studies $[19,20,29,30,33-35]$ to prevent the "pseudo-open bite" due to under-erupted permanent incisors.

Other inclusion criteria considered by few authors were no teeth absence due to ageneses or extractions 
Table 1 Results: trial design, treatment modalities, characteristics of the samples

\begin{tabular}{|c|c|c|c|c|}
\hline $\begin{array}{l}\text { Selected references } \\
\text { Year of publication } \\
\text { Study design }\end{array}$ & Treatment modalities & Sample size & Age (years) & Sex \\
\hline Albogha H et al. [24] & RMI vs. MBB & 15 (RMI) & 8.1-13.5 (RMI) & $4 \mathrm{M}, 11 \mathrm{~F}(\mathrm{RMI})$ \\
\hline 2015 & & 15 (MBB) & 8.5-13.5 (MBB) & $6 \mathrm{M}, 9 \mathrm{~F}(\mathrm{MBB})$ \\
\hline \multicolumn{5}{|l|}{$P, L, C T$} \\
\hline Mucedero M et al. [35] & Q-H/C vs. UCG & $28(\mathrm{Q}-\mathrm{H} / \mathrm{C})$ & $8.2 \pm 1.3(\mathrm{Q}-\mathrm{H} / \mathrm{C})$ & $11 \mathrm{M}, 7 \mathrm{~F}(\mathrm{Q}-\mathrm{H} / \mathrm{C})$ \\
\hline 2013 & & 20 (UCG) & $8.1 \pm 0.4(\mathrm{UCG})$ & 10M, 10F (UCG) \\
\hline \multicolumn{5}{|l|}{$\mathrm{R}, \mathrm{L}, \mathrm{CCT}$} \\
\hline Torres FC et al. [33] & $R P C+V C C$ vs. FPC + VCC & $30(\mathrm{RPC}+\mathrm{VCC})$ & $8.33 \pm 0.73(\mathrm{RPC}+\mathrm{VCC})$ & $8 \mathrm{M}, 22 \mathrm{~F}(\mathrm{RPC}+\mathrm{VCC})$ \\
\hline 2012 & & $30(F P C+V C C)$ & $8.54 \pm 0.88(F P C+V C C)$ & $11 \mathrm{M}, 19 \mathrm{~F}(\mathrm{FPC}+\mathrm{VCC})$ \\
\hline \multicolumn{5}{|l|}{$P, L, C T$} \\
\hline Cassis MA et al. [34] & $B S+$ VCC vs. UCG & $30(B S+V C C)$ & $8.14 \pm 0.73(B S+V C C)$ & $9 \mathrm{M}, 21 \mathrm{~F}(\mathrm{BS}+\mathrm{VCC})$ \\
\hline 2012 & & 30 (UCG) & $8.36 \pm 1.05$ (UCG) & $30.5 \mathrm{M}, 25 \mathrm{~F}(\mathrm{UCG})$ \\
\hline \multicolumn{5}{|l|}{$P, L, C C T$} \\
\hline Doshi UH et al. [23] & SLBB vs. MBB & 10 (SLBB) & 8-13(SLBB) & $5 \mathrm{M}, 5 \mathrm{~F}(\mathrm{SLBB})$ \\
\hline 2010 & & 10 (MBB) & 8-13 (MBB) & $3 \mathrm{M}, 7 \mathrm{~F}(\mathrm{MBB})$ \\
\hline$P, L, R C T$ & & $10(U C G)$ & 8-13 (UCG) & \\
\hline Giuntini $\vee$ et al. [32] & $\mathrm{Q}-\mathrm{H} / \mathrm{C}$ vs. RPC & $20(\mathrm{Q}-\mathrm{H} / \mathrm{C})$ & $8.4 \pm 1.4(\mathrm{Q}-\mathrm{H} / \mathrm{C})$ & $5 \mathrm{M}, 15 \mathrm{~F}(\mathrm{Q}-\mathrm{H} / \mathrm{C})$ \\
\hline 2008 & & 20 (RPC) & $8.4 \pm 1(\mathrm{RPC})$ & $9 \mathrm{M}, 11 \mathrm{~F}(\mathrm{RPC})$ \\
\hline \multicolumn{5}{|l|}{$R, L, C T$} \\
\hline \multirow{6}{*}{$\begin{array}{l}\text { Cinsar A et al. [22] } \\
\text { (subgroups) }\end{array}$} & RMI vs. UCG & 10 (RMI) & M $11 \pm 0.4$ & $3 \mathrm{M}, 7 \mathrm{~F}(\mathrm{RMI})$ \\
\hline & & 10 (UCG) & $F 10.3 \pm 0.2$ & $3 \mathrm{M}, 7 \mathrm{~F}(\mathrm{UCG})$ \\
\hline & & & (RMI) & \\
\hline & & & & \\
\hline & & & M $11 \pm 1$ & \\
\hline & & & F $10.8 \pm 0.9$ (UCG) & \\
\hline Defraia E et al. [41] & OBB vs. uCG & 20 (OBB) & $8.2 \pm 0.8(\mathrm{OBB})$ & $11 \mathrm{M}, 9 \mathrm{~F}(\mathrm{OBB})$ \\
\hline 2007 & & 23 (UCG) & $10.8 \pm 1.5($ UCG $)$ & 23 (UCG) \\
\hline \multicolumn{5}{|l|}{$\mathrm{R}, \mathrm{L}, \mathrm{CCT}$} \\
\hline Cozza P et al. [30] & Q-H/C vs. UCG & $21(\mathrm{Q}-\mathrm{H} / \mathrm{C})$ & $8.4 \pm 1.5(\mathrm{Q}-\mathrm{H} / \mathrm{C})$ & $6 \mathrm{M}, 15 \mathrm{~F}(\mathrm{Q}-\mathrm{H} / \mathrm{C})$ \\
\hline 2007 & & $21(U C G)$ & $8.6 \pm 11 \mathrm{M}(\mathrm{UCG})$ & $10 \mathrm{M}, 11 \mathrm{~F}(\mathrm{UCG})$ \\
\hline \multicolumn{5}{|l|}{$\mathrm{R}, \mathrm{L}, \mathrm{CCT}$} \\
\hline Cozza P et al. [31] & Q-H/C vs. OBB & $21(\mathrm{Q}-\mathrm{H} / \mathrm{C})$ & $8.4 \pm 1.4(\mathrm{Q}-\mathrm{H} / \mathrm{C})$ & $6 \mathrm{M}, 15 \mathrm{~F}(\mathrm{Q}-\mathrm{H} / \mathrm{C})$ \\
\hline 2007 & & 20 (OBB) & & \\
\hline \multicolumn{5}{|l|}{$R, L, C T$} \\
\hline Pedrin F et al. [27] & RPC + VCC vs. UCG & $30(R P C+V C C)$ & $8.61(\mathrm{RPC}+\mathrm{VCC})$ & $10 \mathrm{M}, 20 \mathrm{~F}(\mathrm{RPC}+\mathrm{VCC})$ \\
\hline 2006 & & 30 (UCG) & 8.33 (UCG) & $7 \mathrm{M}, 23 \mathrm{~F}(\mathrm{UCG})$ \\
\hline \multicolumn{5}{|l|}{$P, L, C C T$} \\
\hline Torres F et al. [28] & RPC + VCC vs. UCG & $30(R P C+V C C)$ & $8.33(\mathrm{RPC}+\mathrm{VCC})$ & $8 \mathrm{M}, 22 \mathrm{~F}(\mathrm{RPC}+\mathrm{VCC})$ \\
\hline 2006 & & 30 (UCG) & 8.61 (UCG) & 7M, 23F (UCG) \\
\hline \multicolumn{5}{|l|}{$P, L, R C T$} \\
\hline Cozza P et al. [29] & Q-H/C vs. UCG & $23(\mathrm{Q}-\mathrm{H} / \mathrm{C})$ & $8.4 \pm 1.4(\mathrm{Q}-\mathrm{H} / \mathrm{C})$ & $7 \mathrm{M}, 16 \mathrm{~F}(\mathrm{Q}-\mathrm{H} / \mathrm{C})$ \\
\hline 2006 & & 23 (UCG) & $9.1 \pm 1.6$ (UCG) & 10M, 13F (UCG) \\
\hline \multicolumn{5}{|l|}{$\mathrm{R}, \mathrm{L}, \mathrm{CCT}$} \\
\hline Iscan HN et al. [16] & VCC vs. UCG & 18 (VCC) & 8.08-11.11 (VCC) & $6 \mathrm{M}, 12 \mathrm{~F}(\mathrm{VCC})$ \\
\hline
\end{tabular}


Table 1 Results: trial design, treatment modalities, characteristics of the samples (Continued)

\begin{tabular}{|c|c|c|c|c|}
\hline 2002 & & 17 (UCG) & $8.40-12.26$ (UCG) & $6 \mathrm{M}, 11 \mathrm{~F}(\mathrm{UCG})$ \\
\hline \multicolumn{5}{|l|}{$P, L, C C T$} \\
\hline Bazzucchi A et al. [21] & MBB vs. UCG & 29 (MBB) & $11.08 \pm 3.08(\mathrm{MBB})$ & $6 \mathrm{M}, 23 \mathrm{~F}(\mathrm{MBB})$ \\
\hline 1999 & & 29 (UCG) & $11 \pm 3.08$ (UCG) & $6 \mathrm{M}, 23 \mathrm{~F}(\mathrm{UCG})$ \\
\hline \multicolumn{5}{|l|}{$R, L, C C T$} \\
\hline Iscan HN and Sarisoy L [20] & PBB5 vs. PBB10 vs. UCG & 13 (PBB5) & 8.9-13.5 (PBB5) & $4 \mathrm{M}, 9 \mathrm{~F}(\mathrm{PBB} 5)$ \\
\hline 1997 & & 12 (PBB10) & 8.7-14.5 (PBB10) & 3M, 9F (PBB10) \\
\hline$P, L, C C T$ & & 14 (UCG) & 8.9-13.6 (UCG) & $3 \mathrm{M}, 11 \mathrm{~F}(\mathrm{UCG})$ \\
\hline Erbay E et al. [40] & $F R+$ LSE vs. UCG & $20(F R+L S E)$ & $8.7 \pm 0.5(\mathrm{OBB})$ & $7 \mathrm{M}, 13 \mathrm{~F}(\mathrm{FR}+\mathrm{LSE})$ \\
\hline 1995 & & 20 (UCG) & $8.9 \pm 1.2(\mathrm{UCG})$ & 7M, 13F (UCG) \\
\hline \multicolumn{5}{|l|}{$P, L, R C T$} \\
\hline Iscan HN et al. [19] & SLBB vs. PBB + VCC & 11 (SLBB) & 8.62-13.54 (SLBB) & Not declared \\
\hline 1992 & & $12(\mathrm{PBB}+\mathrm{VCC})$ & 7.39-11.67 (PBB + VCC) & \\
\hline \multicolumn{5}{|l|}{$R, L, C T$} \\
\hline Weinbach JR and Smith RJ. [39] & OBB vs. UCG & 26 (OBB) & $7.08-12.88$ & $27 \mathrm{M}, 12 \mathrm{~F}$ \\
\hline 1992 & & $13(\mathrm{OBB}+\mathrm{HPH})$ & & \\
\hline$R, L, C C T$ & & Published normal growth standards & & \\
\hline Kuster R and Ingervall B [18] & SLBB vs. MBB & 22 (SLBB) & 7.4-11.56(SLBB) & $11 \mathrm{M}, 11 \mathrm{~F}(\mathrm{SLBB})$ \\
\hline 1992 & & 11 (MBB) & 9.72-14.4 (MBB) & $4 \mathrm{M}, 7 \mathrm{~F}(\mathrm{MBB})$ \\
\hline \multicolumn{5}{|l|}{$R, L, C T$} \\
\hline Ngan P et al. [38] & A-HPH vs. UCG & $8(\mathrm{~A}-\mathrm{HPH})$ & 10.24(A-HPH) & $2 \mathrm{M}, 6 \mathrm{~F}$ \\
\hline 1992 & & 8 (UCG) & 10.24 (UCG) & \\
\hline \multicolumn{5}{|l|}{$R, L, C C T$} \\
\hline Haydar B and Enacar A [37] & $F R+L S E$ vs. UCG & $11(F R)$ & $8.8 \pm 1.17$ (OBB) & Not available \\
\hline 1992 & & 10 (UCG) & $8.3 \pm 1.06$ (UCG) & \\
\hline \multicolumn{5}{|l|}{$P, L, C C T$} \\
\hline Kiliaridis S et al. [17] & MBB vs. PBB & 10 (MBB) & $8.9-16.1$ & $3 \mathrm{M}, 7 \mathrm{~F}(\mathrm{MBB})$ \\
\hline 1990 & & 10 (PBB) & & $3 \mathrm{M}, 7 \mathrm{~F}$ (PBB) \\
\hline \multicolumn{5}{|l|}{$P, L, C T$} \\
\hline Frankel R [36] & $F R+L S E$ vs. UCG & $30(F R)$ & 7 (FR) & Not declared \\
\hline 1983 & & 11 (UCG) & 8 (UCG) & \\
\hline$R, L, C C T$ & & & & \\
\hline
\end{tabular}

[19, 33-35, 40], no previous orthodontic treatment [33], no crowding [27, 28, 33, 34], no need for adenoidectomy or tonsillectomy [16, 19, 20, 28], excessive overjet [38], anterior open bite unchanged or increased in the last 6 months [17], large interlabial distance, and postural weakness of the orofacial muscles [36]. These aspects were not examined by the other authors.

Most of the trials selected patients with anterior open bite regardless their skeletal and molar class.

Details about sample size, age, and sex of the participants were resumed in Table 1.
Sexual dimorphism was evaluated and not found in three studies $[27,28,34]$ while it was found for few parameters in one study [40].

\section{Success rate}

The success rate was $100 \%$ in four studies [17, 19, 22, 38], $80-90 \%$ in eight studies [16, 27-30, 34, 35, 41], and $67 \%$ in one study [39] with untreated control group (Table 2).

Seven studies omitted the success rate $[18,21,23,31$, $36,37,40]$. 
Table 2 Results: success rate, treatment duration, reduction of open bite and divergency, side effects and stability

\begin{tabular}{|c|c|c|c|c|c|c|c|}
\hline $\begin{array}{l}\text { Selected } \\
\text { references }\end{array}$ & Success rate & Treatment duration/observation time & $\begin{array}{l}\text { Time of daily } \\
\text { appliance wear } \\
\text { (h) }\end{array}$ & $\begin{array}{l}\text { Reduction of open } \\
\text { bite and } \\
\text { divergency }\end{array}$ & Methods of measurement & Side effects & Follow-up/stability \\
\hline \multirow{2}{*}{$\begin{array}{l}\text { Albogha H } \\
\text { et al. [24] }\end{array}$} & $33 \%(\mathrm{RMI})$ & \multirow[t]{2}{*}{4 months } & \multirow[t]{2}{*}{$24 \mathrm{~h}$} & Yes open bite & \multirow[t]{2}{*}{ Cephalometry } & \multirow{2}{*}{$\begin{array}{l}\text { Both hindered } \\
\text { oral hygiene. }\end{array}$} & \multirow{2}{*}{$\begin{array}{l}\text { No/no stability } \\
\text { information }\end{array}$} \\
\hline & $27 \%$ (MBB) & & & Yes divergency & & & \\
\hline \multirow{2}{*}{$\begin{array}{l}\text { Mucedero M } \\
\text { et al. [35] }\end{array}$} & \multirow{2}{*}{$86 \%$} & \multirow[t]{2}{*}{18 months/no retention information } & \multirow[t]{2}{*}{$24 \mathrm{~h}$} & Yes open bite & \multirow[t]{2}{*}{ Cephalometry } & \multirow[t]{2}{*}{ No } & \multirow{2}{*}{$\begin{array}{l}\text { At least } 5 \text { years/no relapse } \\
\text { (data not suitable with } \\
\text { inclusion criteria) }\end{array}$} \\
\hline & & & & Yes divergency & & & \\
\hline \multirow[t]{2}{*}{$\begin{array}{l}\text { Torres FC et } \\
\text { al. [33] }\end{array}$} & $\begin{array}{l}70 \%(F P C+ \\
\text { VCC) }\end{array}$ & 12 months/no & $24 \mathrm{~h}(\mathrm{RPC}, \mathrm{FPC})$ & Yes open bite & \multirow[t]{2}{*}{ Cephalometry } & \multirow[t]{2}{*}{ No } & \multirow[t]{2}{*}{$\begin{array}{l}\text { No/no stability } \\
\text { information }\end{array}$} \\
\hline & $\begin{array}{l}50 \%(\mathrm{RPC}+ \\
\text { VCC) }\end{array}$ & Retention information & 14-16 h (VCC) & No divergency & & & \\
\hline \multirow{2}{*}{$\begin{array}{l}\text { Cassis MA } \\
\text { et al. [34] }\end{array}$} & \multirow[t]{2}{*}{$86.7 \%$} & \multirow[t]{2}{*}{12 months/no retention information } & $24 \mathrm{~h}(\mathrm{BS})$ & Yes open bite & \multirow[t]{2}{*}{ Cephalometry } & \multirow[t]{2}{*}{ No } & \multirow{2}{*}{$\begin{array}{l}\text { No/no stability } \\
\text { information }\end{array}$} \\
\hline & & & 14-16 h (VCC) & Yes divergency & & & \\
\hline \multirow{2}{*}{$\begin{array}{l}\text { Doshi UH et } \\
\text { al. [23] }\end{array}$} & \multirow{2}{*}{ Not declared } & \multirow{2}{*}{$\begin{array}{l}\text { Until an edge-to-edge bite was achieved } \\
\text { (max } 8 \text { months)/retention with passive BB } \\
\text { for } 10 \text { months }\end{array}$} & \multirow[t]{2}{*}{ not declared } & Yes open bite & \multirow{2}{*}{$\begin{array}{l}\text { Clinical evaluation, cephalometry, } \\
\text { electromyography }\end{array}$} & \multirow{2}{*}{$\begin{array}{l}\text { Broken spring } \\
\text { replaced in } 7 \\
\text { pz (SLBB) }\end{array}$} & \multirow{2}{*}{$\begin{array}{l}10 \text { months/insignificant } \\
\text { dentoalveolar relapse }\end{array}$} \\
\hline & & & & Yes divergency & & & \\
\hline \multirow{2}{*}{$\begin{array}{l}\text { Giuntini V et } \\
\text { al. [32] }\end{array}$} & $90 \%(\mathrm{Q}-\mathrm{H} / \mathrm{C})$ & 18 months/no retention information & $24 \mathrm{~h}(\mathrm{Q}-\mathrm{H} / \mathrm{C})$ & Yes open bite & Cephalometry & No & No/no stability \\
\hline & $60 \%(\mathrm{RPC})$ & & $16 \mathrm{~h}(\mathrm{RPC})$ & Yes divergency & & & \\
\hline Cinsar A et & $100 \%$ & 9-11 months/no retention information & $24 \mathrm{~h}$ & Yes open bite & Cephalometry & No & No/no stability \\
\hline $\begin{array}{l}\text { al. [2L] } \\
\text { (subgroups) }\end{array}$ & & & & Yes divergency & & & Informatıon \\
\hline Defraia E et & $85 \%$ & 18 months/about 12 months of retention & $24 \mathrm{~h}$ & Yes open bite & Cephalometry & No & No/no stability \\
\hline & & & & Yes divergency & & & \\
\hline Cozza P et & $85 \%$ & 18 months/no retention (16 patients); & $24 \mathrm{~h}$ & Yes open bite & Cephalometry & No & 2 years/relapse in $15 \%$ of \\
\hline & & $\begin{array}{l}\text { removable appliance for retention for } \\
1 \text { year (5 patients) }\end{array}$ & & Yes divergency & & & \\
\hline Cozza P et & Not declared & Active treatment & $24 \mathrm{~h}(\mathrm{Q}-\mathrm{H} / \mathrm{C})$ & Yes open bite & Cephalometry & No & 1 year/no relapse $(\mathrm{QH} / \mathrm{C})$ \\
\hline & & 18 months/no retention (Q-H/C) & $24 \mathrm{~h}(\mathrm{OBB})$ & Yes divergency & & & No stability information \\
\hline & & $\begin{array}{l}18 \text { months/no retention, with the } \\
\text { exception of a few patients who continued } \\
\text { to use the OBB at night (OBB) }\end{array}$ & & OBB) & & & \\
\hline & & Observation time & & & & & \\
\hline & & 2.6 years \pm 9 months $(\mathrm{Q}-\mathrm{H} / \mathrm{C})$ & & & & & \\
\hline & & 2.5 years \pm 1.2 years (UCG) & & & & & \\
\hline Pedrin F et & $80 \%$ & 12 months/no retention information & $14-16 \mathrm{~h}$ & Yes open bite & Cephalometry & No & No/no stability \\
\hline & & & & No divergency & & & \\
\hline Torres F et & $80 \%$ & 12 months/no retention information & $14-16 \mathrm{~h}$ & Yes open bite & Cephalometry & No & No/no stability \\
\hline & & & & No divergency & & & \\
\hline
\end{tabular}


Table 2 Results: success rate, treatment duration, reduction of open bite and divergency, side effects and stability (Continued)

\begin{tabular}{|c|c|c|c|c|c|c|c|}
\hline \multirow{2}{*}{$\begin{array}{l}\text { Cozza P et } \\
\text { al. [29] }\end{array}$} & \multirow[t]{2}{*}{$90 \%$} & \multirow[t]{2}{*}{18 months/no retention information } & \multirow[t]{2}{*}{$24 \mathrm{~h}$} & Yes open bite & \multirow[t]{2}{*}{ Cephalometry } & \multirow[t]{2}{*}{ No } & \multirow{2}{*}{$\begin{array}{l}\text { No/No stability } \\
\text { information }\end{array}$} \\
\hline & & & & Yes divergency & & & \\
\hline \multirow{2}{*}{$\begin{array}{l}\text { Işcan HN et } \\
\text { al. [16] }\end{array}$} & \multirow[t]{2}{*}{$88 \%$} & \multirow{2}{*}{$\begin{array}{l}6-12 \text { months until overbite was obtained } \\
\text { (mean } 9 \text { months) no retention }\end{array}$} & \multirow[t]{2}{*}{$16 \mathrm{~h}$} & Yes open bite & \multirow[t]{2}{*}{ Cephalometry } & \multirow[t]{2}{*}{ No } & \multirow{2}{*}{$\begin{array}{l}\text { No/no stability } \\
\text { information }\end{array}$} \\
\hline & & & & Yes divergency & & & \\
\hline \multirow{3}{*}{$\begin{array}{l}\text { Bazzucchi A } \\
\text { et al. [21] }\end{array}$} & \multirow[t]{3}{*}{ Not declared } & 8 months (MBB) & \multirow[t]{3}{*}{ Not declared } & Yes openbite & \multirow[t]{3}{*}{ Cephalometry } & \multirow[t]{3}{*}{ No } & \multirow{3}{*}{$\begin{array}{l}\text { Not suitable with } \\
\text { inclusion criteria }\end{array}$} \\
\hline & & 9 months (uCG) & & Yes divergency & & & \\
\hline & & No retention information & & $\begin{array}{l}\text { (Not statistically } \\
\text { but clinically } \\
\text { significant } \\
\text { changes) }\end{array}$ & & & \\
\hline \multirow{5}{*}{$\begin{array}{l}\text { Işcan HN } \\
\text { and Sarisoy } \\
\text { L [20] }\end{array}$} & $80 \%$ (PBB5) & \multirow{2}{*}{$\begin{array}{l}4-10 \text { months, until an overbite of } 1- \\
1.5 \mathrm{~mm} \text { was achieved (PBB5) }\end{array}$} & \multirow[t]{5}{*}{$18 \mathrm{~h}$} & Yes open bite & \multirow[t]{5}{*}{ Cephalometry } & \multirow[t]{5}{*}{ No } & \multirow{5}{*}{$\begin{array}{l}\text { No/no stability } \\
\text { information }\end{array}$} \\
\hline & $66 \%$ (PBB10) & & & Yes divergency & & & \\
\hline & & $\begin{array}{l}4-13 \text { months, until an overbite of 1- } \\
1.5 \mathrm{~mm} \text { was achieved (PBB10) }\end{array}$ & & & & & \\
\hline & & 7-9 months (UCG) & & & & & \\
\hline & & No retention information & & & & & \\
\hline \multirow{3}{*}{$\begin{array}{l}\text { Erbay E et } \\
\text { al. [40] }\end{array}$} & \multirow[t]{3}{*}{ Not declared } & 24 months (FR) & \multirow[t]{3}{*}{$18 \mathrm{~h}$} & Yes open bite & \multirow[t]{3}{*}{ Cephalometry } & \multirow[t]{3}{*}{ No } & No/no stability \\
\hline & & 24 months (UCG) & & Yes divergency & & & \\
\hline & & No retention information & & & & & \\
\hline Işcan HN et & $100 \%$ & $1-10$ months until an overbite of $1-$ & $16 \mathrm{~h}$ & Yes open bite & Cephalometry & No & No/no stability \\
\hline & & $1.5 \mathrm{~mm}$ was achieved (SLBB) & & Yes divergency & & & information \\
\hline & & $\begin{array}{l}3-9 \text { months until an overbite of 1-1.5 mm } \\
\text { was achieved (PBB) then worn only at } \\
\text { night for retention }\end{array}$ & & & & & \\
\hline Weinbach & $67 \%$ had a & Mean 20 months & Not declared & Yes open bite & Cephalometry & No & No/no stability \\
\hline $\begin{array}{l}\text { JR and } \\
\text { Smith RJ } \\
\text { [39] }\end{array}$ & $\begin{array}{l}\text { reduction of } \\
\text { open bite }\end{array}$ & No retention & & Yes divergency & & & information \\
\hline Kuster R & Not declared & SLBB 1 year & At night (SLBB) & Yes open bite & Bite force, cephalometry, & Broken spring & 6 months/tendency to \\
\hline $\begin{array}{l}\text { and } \\
\text { Ingervall B }\end{array}$ & & MBB 3 months & $24 \mathrm{~h}(\mathrm{MBB})$ & Yes divergency & & $\begin{array}{l}\text { replaced in } 12 \\
\text { pz (SLBB) }\end{array}$ & \\
\hline & & 2 MBB patients: no retention & & & & & \\
\hline & & 1 MBB: activator as retention for 1 year & & & & & No stability information \\
\hline & & $\begin{array}{l}3 \text { MBB patients: upper removable plate } \\
\text { with posterior platforms } 6-8 \text { months }\end{array}$ & & & & & (SLBB) \\
\hline & & $\begin{array}{l}3 \text { MBB patients } 1 \text { year multibanded } \\
\text { appliance }\end{array}$ & & & & & \\
\hline Ngan $\mathrm{P}$ et & $100 \%$ & Mean 14 months until overcorrection of & 2 h (first 3 days) & Yes open bite & Cephalometry & No & No/no stability \\
\hline & & dental and skeletal relationsnip & $\begin{array}{l}\text { than increased } \\
\text { until } 24 \mathrm{~h}(\mathrm{~A})\end{array}$ & Yes divergency & Study casts & & \\
\hline
\end{tabular}


Table 2 Results: success rate, treatment duration, reduction of open bite and divergency, side effects and stability (Continued)

\begin{tabular}{|c|c|c|c|c|c|c|c|}
\hline & & No retention & 12-14 h (HPH) & & & & \\
\hline \multirow{2}{*}{$\begin{array}{l}\text { Haydar B } \\
\text { and Enacar } \\
\text { A [37] }\end{array}$} & \multirow[t]{2}{*}{ Not declared } & FR 1235 years & \multirow[t]{2}{*}{ Not available } & Yes open bite & \multirow[t]{2}{*}{ Cephalometry } & \multirow[t]{2}{*}{ No } & \multirow{2}{*}{$\begin{array}{l}\text { No/no stability } \\
\text { information }\end{array}$} \\
\hline & & UCG 1024 years & & No divergency & & & \\
\hline \multirow{3}{*}{$\begin{array}{l}\text { Kiliaridis S et } \\
\text { al. [17] }\end{array}$} & \multirow{3}{*}{$100 \%$} & 6 months & \multirow[t]{3}{*}{$18 \mathrm{~h}$} & Yes open bite & \multirow{3}{*}{$\begin{array}{l}\text { Cephalometry, study casts, intra-oral } \\
\text { photographs, monthly analysis of the } \\
\text { stomatognatic system }\end{array}$} & Lateral & \multirow{3}{*}{$\begin{array}{l}\text { No/no stability } \\
\text { information }\end{array}$} \\
\hline & & \multirow[t]{2}{*}{ No retention } & & \multirow{2}{*}{ Yes divergency } & & ) & \\
\hline & & & & & & $\begin{array}{l}\text { Effect declined } \\
\text { with time } \\
\text { (PBB) }\end{array}$ & \\
\hline \multirow[t]{3}{*}{$\begin{array}{l}\text { Fränkel R et } \\
\text { al. [36] }\end{array}$} & \multirow[t]{3}{*}{ Not declared } & $\begin{array}{l}\text { No treatment and retention durations } \\
\text { information }\end{array}$ & \multirow[t]{3}{*}{ Not declared } & Yes open bite & \multirow[t]{3}{*}{ Cephalometry } & \multirow[t]{3}{*}{ No } & $\begin{array}{l}\text { At least } 4 \text { years out of } \\
\text { retention/Stability if lips }\end{array}$ \\
\hline & & Observation time & & Yes divergency & & & $\begin{array}{l}\text { Sealed without muscular } \\
\text { straint. }\end{array}$ \\
\hline & & 8 years & & & & & Relapse rate not declared \\
\hline
\end{tabular}

loaded bite block, OBB open bite bionator, $P B B 5$ posterior bite blocks $5 \mathrm{~mm}, P B B 10$ posterior bite blocks $10 \mathrm{~mm}$, FR Fränkel appliance, LSE lip seal exercises, $A$-HPH Teuscher appliance 
A $20-30 \%$ difference in the success rate was found in two studies which compared two different treatment modalities [32, 33]. Lower differences were found in two studies [20, 24].

\section{Treatment duration and open-bite reduction}

The treatment duration varied significantly among the different study protocols (Table 2), and it was not declared in one study [36].

All the trials observed a reduction of the open bite in the treatment group. Bazzucchi [21] reported no statistically significant changes between treated subjects and controls even if dental and skeletal changes were found to be clinically relevant.

In 20 studies, the treatment had also skeletal effects [16-24, 29-32, 34-36, 38-41].

The amount of open-bite reduction varied from 3.1 to $5.1 \mathrm{~mm}$ for RPC, alone [32] or in association with VCC [27, 33], and from 4.1 to $5.44 \mathrm{~mm}$ for fixed cribs as FPC [33], Q-H/C [29-31, 35], BS [34] with [33, 34] or without [30-32, 35] VCC.

Isscan found that the $\mathrm{VCC}$ alone produced $3.92 \mathrm{~mm}$ of overbite correction [16].

The mean correction of the overbite achieved with bite blocks varied from 2.25 to $4.58 \mathrm{~mm}$ for PBB [19, 20, 37], from 1.3 to $3.59 \mathrm{~mm}$ for SLBB [18, 19, 23], from 2.00 to $4.9 \mathrm{~mm}$ for $\mathrm{MBB}[17,21,23,24,35]$, and from 3.1 to 4.55 for RMI $[22,24]$.

OBB showed a mean correction of overbite varying from 1.3 to $2.7 \mathrm{~mm}$.

FR was used in three studies [36, 37, 40] which reported a reduction of the overbite varying from 2.63 to $5 \mathrm{~mm}$ due to the therapy.

The only trial about A-HPH [38] did not declare the amount of correction of the open bite (Table 3 ).

\section{Side effects and costs}

Regarding side effects, one study declared that RMI and MBB hindered oral hygiene [24].

Although no spurs were lost during the treatment period, Cassis reported that the possibility to fall and being aspired into the lungs or swallowed should be considered in the appliance selection [34].

In two trials $[18,23]$, more than half SLBBs were broken during the treatment.

One study reported that unilateral crossbite occurred in half of the patients who wore MBB extensively [17].

The disadvantage of the $\mathrm{PBB}$ is that treatment effects declined over time, possibly because of a decrease in the force applied to the antagonist teeth by the mandibular elevator muscles [19].

Işcan found that increasing the height of PBB resulted in an increase in the gonial angle probably because of a muscular response to the artificially increased vertical dimension and suggested that this angle should be examined in the long term [20].

One study reported that FR appliance caused an unexpected backward rotation of the mandible in the treated group [37].

No studies performed a cost analysis.

\section{Stability}

Eighteen studies did not analyze treatment stability [16, 17, 19-22, 24, 27-29, 32-34, 37-41]. Three studies found insignificant or absent relapse [23, 31, 35].

Mucedero [35] reported stability after at least 5 years from the end of the treatment. These data are not suitable with our inclusion criteria since a fixed appliance was used during the follow-up period.

Cozza [30] evaluated the treated group of a previous study [29] 2 years after the active treatment finding relapse in $15 \%$ of the subjects.

Kuster and Ingervall [18] did not provide stability information about the treatment with SLBB, while they reported a tendency to relapse for $\mathrm{MBB}$ group after 1 year. Fränkel [36] reported that when open bite was associated with an hyperdivergent skeletal pattern, relapse occurred in all treated cases unless a competent anterior oral seal had been achieved.

However, these last two studies did not declare the relapse rate (Table 3 ).

\section{Quality analysis}

Since several items required in quality reviews [46, 47] were not applicable to this study, the quality of the articles was judged as low, medium, or high as proposed by Petrén et al [48].

Most studies presented shortcomings, problems of selection, and misuration bias.

Research quality was low in ten studies [16, 17, 19, 21, $23,24,27,29,38,39$ ] and medium in 14 (Table 3).

Due to the insufficient number of RCTs, the lack of standardization of diagnostic criteria, inclusion criteria, validity measures to evaluate outcomes, and methodological limitations, a meta-analysis could not be performed.

\section{Discussion}

Recently, Feres et al. [42] performed a systematic review on the effectiveness of the open-bite treatment in growing children and adolescents concluding that consistent results were not found. A further review by LentiniOliveira published in 2014 including only RCTs assessed that there were no clear evidence on which to make a clinical decision of the type of intervention to use [43].

Besides, the present study aims to focus wholly on the open-bite treatment of subjects in the mixed dentition. 
Table 3 Quality analysis

\begin{tabular}{|c|c|c|c|c|c|c|c|c|}
\hline Article & $\begin{array}{l}\text { Previous estimate } \\
\text { of sample size }\end{array}$ & $\begin{array}{l}\text { Selection } \\
\text { description }\end{array}$ & Withdrawals & $\begin{array}{l}\text { Valid } \\
\text { method }\end{array}$ & $\begin{array}{l}\text { Method error } \\
\text { analysis }\end{array}$ & $\begin{array}{l}\text { Blinding in } \\
\text { measurements }\end{array}$ & $\begin{array}{l}\text { Adequate statistics } \\
\text { provided }\end{array}$ & $\begin{array}{l}\text { Judge quality } \\
\text { standard }\end{array}$ \\
\hline Albogha H et al. [24] & Not & Adequate & Not known & Partly & Yes & Not & Yes & Low \\
\hline Mucedero M et al. [35] & No/not known & Adequate & Not known & Yes & Yes & Not & Yes & Medium \\
\hline Torres FC et al. [33] & No/not known & Adequate & Not known & Yes & Yes & Not & Yes & Medium \\
\hline Cassis MA et al. [34] & Yes & Adequate & Not known & Yes & Yes & Not & Yes & Medium \\
\hline Doshi UH et al. [23] & Not & Adequate & Not known & Partly & Yes & Not & No & Low \\
\hline Giuntini V et al. [32] & Not & Adequate & Not known & Yes & Yes & Not & Yes & Medium \\
\hline $\begin{array}{l}\text { Cinsar A et al. [22] } \\
\text { subgroup. }\end{array}$ & Not & Adequate & Not known & Yes & Yes & Not & Yes & Medium \\
\hline Defraia E et al. [41] & Not & Adequate & Not known & Yes & Yes & Not & Yes & Medium \\
\hline Cozza P et al. [30] & Not & Adequate & Not known & Yes & Yes & Not & Yes & Medium \\
\hline Cozza P et al. [31] & Not & Adequate & Not known & Yes & Yes & Not & Yes & Medium \\
\hline Pedrin F et al [27] & Not & Adequate & None & Partly & Yes & Not & Not & Low \\
\hline Torres FC et al. [28] & Yes & Adequate & Not known & Partly & Yes & Not & Not & Medium \\
\hline Cozza P et al. [29] & Not & Adequate & Not known & Partly & Yes & Not & Yes & Low \\
\hline Işcan HN et al. [19] & Not & Adequate & Not known & Yes & Not & Not & Not & Low \\
\hline Bazzucchi A et al. [21] & Not & Adequate & Not known & Not & Yes & Not & Not & Low \\
\hline $\begin{array}{l}\text { Işcan HN and Sarisoy L } \\
\text { [20] }\end{array}$ & Not & Adequate & Not known & Partly & Yes & Not & Yes & Medium \\
\hline Erbay E et al. [40] & Not & Adequate & Not known & Partly & Not & Not & Yes & Medium \\
\hline Işcan HN et al. [19] & Not & Adequate & One & Yes & Yes & Not & Inadequate & Low \\
\hline $\begin{array}{l}\text { Weinbach JR and Smith RJ } \\
\text { [39] }\end{array}$ & Not & Adequate & Not known & Not & Not & Not & Inadequate & Low \\
\hline $\begin{array}{l}\text { Kuster R and Ingervall B } \\
\text { [18] }\end{array}$ & Not & Adequate & One & Yes & Yes & Not & Yes & Medium \\
\hline Ngan P et al. [38] & Not & Adequate & Not known & Yes & Yes & Not & Inadequate & Low \\
\hline $\begin{array}{l}\text { Haydar B and Enacar A } \\
\text { [37] }\end{array}$ & Not & Adequate & Not known & Partly & Not & Not & Yes & Medium \\
\hline Kiliaridis S et al. [17] & Not & Adequate & Four & Partly & Yes & Yes & Absent & Low \\
\hline Fränkel R [36] & Not & Adequate & Not known & Partly & Not & Not & Yes & Medium \\
\hline
\end{tabular}

The authors included also not RCTs, since in their opinion, their analysis could lead to significant outcomes.

Several treatment modalities were studied and their effects are summarized in Table 4.

The analysis of the results suggests that early treatment was able to intercept and reduce dentoskeletal open bite, in particular when it was caused by an altered function.

VCC alone [16] or associated with other devices [27, $28,33,34]$ produced an increase of the overbite. Although the same protocol of use and similar samples in the studies, some authors $[27,28]$ showed that VCC did not yield favorable skeletal effects, and others [16, 34] reported vertical control and decreased gonial angle probably because of greater compliance.

For the same reason, fixed palatal cribs (FPC; Q-H/C) showed a greater amount of overbite improvement compared to removable appliances [31-33].
On the other hand, RPC + VCC produced a greater improvement in overjet as a result of activations and adjustments.

The therapy with RPC depends on the patient compliance, but, in many cases, it provides a greater comfort than the FPC because it can be worn gradually and can be removed for meals and oral hygiene, which would be favorable from the psychological point of view.

Cribs were found to produce a clinically significant improvement in the maxillomandibular vertical relationships by some authors [29-32, 34, 35], while others [27, 28 , 33] reported only dental effects.

OBB showed an improvement of intermaxillary vertical relationships [31, 39, 41] even if less than Q-H/C [31] and proved to be useful for class II open-bite patients since it reduced facial convexity, ANB angle, and overjet and restricted maxillary molar extrusion, 
Table 4 Summary of effects

\begin{tabular}{|c|c|c|c|c|}
\hline \multicolumn{2}{|c|}{ Treatment modalities } & Summary of effects & $\begin{array}{l}\text { Reduction of } \\
\text { open bite }\end{array}$ & $\begin{array}{l}\text { Reduction of } \\
\text { divergency }\end{array}$ \\
\hline \multirow{2}{*}{\multicolumn{2}{|c|}{ Rapid molar intruder (RMI) }} & Molar intrusion & \multirow[t]{2}{*}{ Yes } & \multirow[t]{2}{*}{ Yes } \\
\hline & & Mandibular autorotation & & \\
\hline \multirow{16}{*}{$\begin{array}{l}\text { Bite } \\
\text { blocks }\end{array}$} & \multirow[t]{6}{*}{ Magnetic bite block (MBB) } & Incisors extrusion, molar intrusion & \multirow[t]{6}{*}{ Yes } & \multirow[t]{6}{*}{ Yes } \\
\hline & & Control of mandibular skeletal height & & \\
\hline & & Mandibular autorotation & & \\
\hline & & Lateral crossbite & & \\
\hline & & More effective than spring loaded bite blocks & & \\
\hline & & Faster and more effective than acrylic bite blocks & & \\
\hline & \multirow[t]{5}{*}{ Spring-loaded bite block (SLBB) } & Incisors extrusion, maxillary molar intrusion & \multirow[t]{5}{*}{ Yes } & \multirow[t]{5}{*}{ Yes } \\
\hline & & Control of posterior dentoalveolar height & & \\
\hline & & Mandibular autorotation & & \\
\hline & & Tendency to break & & \\
\hline & & $\begin{array}{l}\text { Greater ramal inclination and molar intrusion than } \\
\text { acrylic bite blocks }\end{array}$ & & \\
\hline & \multirow{5}{*}{$\begin{array}{l}\text { Posterior bite blocks } 5 \mathrm{~mm} \text { (PBB5); posterior bite } \\
\text { blocks } 10 \mathrm{~mm} \text { (PBB10) }\end{array}$} & Incisive extrusion and lingual tipping, molar intrusion & \multirow[t]{5}{*}{ Yes } & \multirow[t]{5}{*}{ Yes } \\
\hline & & Control of posterior dentoalveolar height & & \\
\hline & & Mandibular autorotation & & \\
\hline & & PBB5 and PBB10 are both effective & & \\
\hline & & $\begin{array}{l}\text { PBB10 produce greater mandibular sagittal growth } \\
\text { and autorotation, increase of gonial angle }\end{array}$ & & \\
\hline \multirow{4}{*}{\multicolumn{2}{|c|}{ Quad-helix/crib (Q-H/C) }} & Stop sucking habits & \multirow[t]{4}{*}{ Yes } & \multirow[t]{4}{*}{ Yes } \\
\hline & & Incisors extrusion and lingual tipping & & \\
\hline & & $\begin{array}{l}\text { More efficient than removable cribs since it does } \\
\text { not need for compliance }\end{array}$ & & \\
\hline & & $\begin{array}{l}\text { Downward rotation of palatal plane and improvement } \\
\text { of intermaxillary vertical relationships }\end{array}$ & & \\
\hline \multirow[t]{5}{*}{$\begin{array}{l}\text { Cribs or } \\
\text { spurs }\end{array}$} & Fixed palatal crib (FPC) & $\begin{array}{l}\text { More efficient than removable cribs since it does not } \\
\text { need for compliance }\end{array}$ & Yes & $\begin{array}{l}\text { Data in } \\
\text { disagreement }\end{array}$ \\
\hline & \multirow[t]{3}{*}{ Removable palatal crib (RPC) } & $\begin{array}{l}\text { Just anterior dento-alveolar effects (extrusion and } \\
\text { verticalization of maxillary and mandibular incisors) }\end{array}$ & \multirow[t]{3}{*}{ Yes } & \multirow[t]{3}{*}{$\begin{array}{l}\text { Data in } \\
\text { disagreement }\end{array}$} \\
\hline & & Molar eruption not controlled & & \\
\hline & & Skeletal effects depend on patient's compliance & & \\
\hline & Spurs (BS) & Dentoalveolar effects & Yes & Yes \\
\hline \multirow{3}{*}{\multicolumn{2}{|c|}{ Vertical chin cup (VCC) }} & Reduction of open bite & \multirow[t]{3}{*}{ Yes } & \multirow{3}{*}{$\begin{array}{l}\text { Data in } \\
\text { disagreement }\end{array}$} \\
\hline & & Molar eruption not controlled & & \\
\hline & & Skeletal effects depend on patient's compliance & & \\
\hline \multirow{7}{*}{$\begin{array}{l}\text { Functional } \\
\text { appliances }\end{array}$} & \multirow[t]{3}{*}{ Open bite bionator (OBB) } & Useful for class II open bite malocclusions & \multirow[t]{3}{*}{ Yes } & \multirow[t]{3}{*}{ Yes } \\
\hline & & Control of maxillary molars extrusion & & \\
\hline & & Improvement of intermaxillary vertical relationships & & \\
\hline & Fränkel appliance + lip seal exercises & Dentoalveolar effects, upper incisors lingual tipping & Yes & Data in \\
\hline & $(F R+L S E)$ & Stability if lips sealed without muscular straint & & aisagreement \\
\hline & & Data about skeletal effects are in disagreement & & \\
\hline & Teuscher appliance (A-HPH) & Effective for class II open bite malocclusions & Yes & Yes \\
\hline
\end{tabular}


achieving vertical control. The association with a $\mathrm{HPH}$ had no significant effect compared with the bionator alone [39].

Bite blocks were found to improve the divergency except for the SLBB tested by Kuster and Ingervall which had just dental effects [18].

For the $\mathrm{PBB}$, the mean change in overbite was less than $3 \mathrm{~mm}$ when used alone [17, 20], $4.6 \mathrm{~mm}$ when used with VCC [19].

Ișcan [20] found that higher PBB were not more effective in improving overbite compared to shorter PBB, but they had greater favorable effects on the sagittal growth and mandibular anterior rotation.

Işcan [19] also demonstrated that SLBB produced greater ramal inclination and molar intrusion than $\mathrm{PBB}$ + VCC even if the amount of correction of the open bite was smaller. Both therapies led also to upward and forward mandibular autorotation and decrease the anterior facial height.

Due to greater dentoalveolar and skeletal effects, MBB proved to be more effective than SLBB [18, 23], PBB [17], and RMI [24].

The MBB elicited significantly greater decreases in the SNA and ANB angles, maxillary incisor angle, and overjet compared with RMI. This can be attributed to the fact that the deformation of the elastic modules of RMI reduced the applied force over time, while it was consistent for magnets. Patients with MBB had then to apply more muscular tension to achieve a lip seal with greater effects attributable to labial pressure. This suggests that MBB might be preferred for open-bite class II with protrusion of the maxillary incisors [24].

Two studies which tested FR + LSE reported an upward and forward mandibular rotation in the treated group, whereas backward rotation continued in the control sample $[36,40]$. On the contrary, Haydar and Enacar [37] denied favorable skeletal effects and assessed an unexpected slight mandibular posterior rotation.

A-HPH was tested in patients with class II skeletal open bite, and it proved to correct open bite and molar relationships due to both favorable dentoalveolar and skeletal effects [38].

\section{Quality of the studies}

Randomization increases the reliability of a study and allows final differences to be ascribable to the treatment and not to random or systematic errors [49].
Only three RCTs about the early treatment of open bite were available $[23,28,40]$. However, randomization process was not described.

Sample size was judged as adequate in six studies [21, 27-29, 33, 34]. In the others, it was partly sufficient or insufficient implying low power and high risk to achieve insignificant outcomes.

Previous estimation of sample size was done by two authors [28, 34], but only one [34] described how it was calculated.

The selection description was adequate or fair in all studies except one [21].

The number of dropouts was declared in four studies [17-19, 27], and it was low.

All the trials provided a clear description of the type and duration of the intervention.

The methods used to detect the treatment effects were valid in 12 studies [16, 18, 19, 22, 30-35, 38, 41] and partly valid in ten $[17,20,23,24,27-29,36,37,40]$.

Some studies [21, 27, 29, 39] lack of an adequate untreated control group probably due to the difficulty in gathering many patients with open bite or the lack of ethical rationale to leave these patients untreated.

Some trials used patients who refused orthodontic therapy $[16,20,34]$ or longitudinal data of untreated individuals enrolled in published growth studies [21, 29, $30,35,37,41$ ] as control group.

Weinbach [39] compared the treatment with published cephalometric standards [50].

In one study [21], the method was considered not valid since participants of the groups were not matched according to their dento-skeletal characteristics but just according to age and sex. In another one [39], the appliance was not used exclusively in patients with anterior open bite and there was not a valid control group.

Groups examined by Kiliaridis [17] and Doshi [23] had a too wide age range with subjects treated in the permanent dentition; Pedrin [27] considered too wide ranges of open bite and MPA angle. In two studies [20, 29], treated and control subjects did not have the same age at the beginning and were not observed for the same amount of time which could have influenced cephalometric evaluation of changes.

In six studies, two interventions were tested at the same time, e.g., MBB or RMI and LSE [24], crib and 
VCC [27, 28], FR and LSE [36, 37, 40], so the results can be attributed either to one or to the other.

SLBB was reported to break frequently in two studies $[18,23]$. Doshi did not specify if the treatment was stopped or the appliances were replaced [23]. Kuster and Ingervall replaced the appliances to the patients [18].

Kiliaridis [17] interrupted the treatment earlier than planned and changed the experimental design because of side effects. This did not allow to perform statistical evaluations of the results.

The analysis of the stability of treatment results can not be considered adequate in most studies, in fact follow-up periods were too short $[23,30,31,38]$ and some patients wore contentions while others did not $[30,31]$, besides some authors applied multibracket appliances during the follow-up [21,35].

Nineteen studies [17-24, 27-35, 38, 41] included a method error analysis, and only one had blind outcome assessment [17].

Furthermore, five studies declared a power analysis $[24,30,31,34,35]$.

Fourteen studies used proper statistical methods [18, 20, $22,29-37,40,41]$. Among the remaining studies, one did not report any statistics [17], whereas in the others, statistics was inadequate, e.g., parametric tests used in insufficient sample size $[16,19,23,24,39]$, paired $t$ test used improperly to compare changes between groups $[21,27$, 28], and inadequate level of significance [38].

\section{Conclusions}

1. Just three RCTs in early treatment of anterior open bite were available.

2. CCTs and CTs indicated the effectiveness of the treatment of anterior open bite in the mixed dentition in improving the overbite.

3. Twenty studies also reported favorable skeletal effects.

4. Studies showed a lack of standardization, important methodological limitations, and shortcomings. The quality level of the studies was not sufficient to draw any evidence-based conclusions. Thus, these results must be viewed with caution.

To determine which treatment is the most effective for early correction of skeletal open bite with a reliable scientific evidence, RCTs with sufficient sample size and more rigorous methodology are required. Future studies should also evaluate stability with a longer follow-up, as well as analysis of tolerability, costs, side effects of the interventions, and patient satisfaction. Diagnostic criteria for anterior open bite should be standardized, and the interventions should be tested to each type of anterior open bite: skeletal or dental. Besides cephalometric measurements, masticatory, swallowing, respiratory functions, maxillary and mandibular growth and measurements, and facial analysis should be evaluated to test the validity of the interventions.

\section{Authors' contributions}

All authors contributed to the study conception and design. LP and LB performed the literary research, selection of articles, data analysis, and interpretation under the supervision of RF and LL. LP drafted the manuscript and acted as the corresponding author. AC, RS, RF, and LL provided critical revisions. AC and RS supervised development of work, helped in data interpretation and manuscript evaluation. All authors approved the manuscript and this submission.

\section{Competing interests}

The authors declare that they have no competing interests.

\section{Author details}

${ }^{1}$ Division of Orthodontics, Department of Surgical and Morphological Sciences, University of Insubria, Varese, Italy. ${ }^{2}$ Postgraduate School of Orthodontics, University of Ferrara, Ferrara, Italy.

Received: 5 June 2016 Accepted: 24 August 2016

Published online: 19 September 2016

\section{References}

1. Subtelny JD, Sakuda M. Open-bite: diagnosis and treatment. Am J Orthod. 1964:50:337-58.

2. Richardson A. Skeletal factors in anterior open-bite and deep overbite. Am J Orthod. 1969:56:114-27.

3. Cangialosi TJ. Skeletal morphologic features of anterior open bite. Am J Orthod. 1984:85:28-36.

4. Grippaudo C, Oliva B, Greco AL, Sferra S, Deli R. Relationship between vertical facial patterns and dental arch form in class II malocclusion. Prog Orthod. 2013;14:43.

5. Zecca PA, Fastuca R, Beretta M, Caprioglio A, Macchi A. Correlation assessment between three-dimensional facial soft tissue scan and lateral cephalometric radiography in orthodontic diagnosis. Int J Dent. 2016;2016: 1473918.

6. Ngan P, Fields HW. Open bite: a review of etiology and management Pediatr Dent. 1997:19:91-8.

7. Farronato G, Giannini L, Galbiati G, Stabilini SA, Maspero C. Orthodonticsurgical treatment: neuromuscular evaluation in open and deep skeletal bite patients. Prog Orthod. 2013;14:41.

8. Fastuca R, Zecca PA, Caprioglio A. Role of mandibular displacement and airway size in improving breathing after rapid maxillary expansion. Prog Orthod. 2014;15:40.

9. Agarwal SS, Nehra K, Sharma M, Jayan B, Poonia A, Bhattal H. Association between breastfeeding duration, non-nutritive sucking habits and dental arch dimensions in deciduous dentition: a cross-sectional study. Prog Orthod. 2014;15:59.

10. Lopes-Freire GM, Cárdenas ABC, Suarez dev Deza JEE, Ustrell-Torrent JM, Oliveira LB, Boj Quesada Jr JR. Exploring the association between feeding habits, non-nutritive sucking habits, and malocclusions in the deciduous dentition. Prog Orthod. 2015;16:43.

11. Rossi RC, Rossi NJ, Rossi NJC, Yamashita HK, Pignatari SSN. Dentofacial characteristics of oral breathers in different ages: a retrospective casecontrol study. Prog Orthod. 2015;16:23.

12. Majorana A, Bardellini E, Amadori F, Conti G, Polimeni A. Timetable for oral prevention in childhood-developing dentition and oral habits: a current opinion. Prog Orthod. 2015;16:39.

13. Nespoli L, Caprioglio A, Brunetti L, Nosetti L. Obstructive sleep apnea syndrome in childhood. Early Hum Dev. 2013;89:S33-7.

14. Sankey WL, Buschang PH, English J, Owen AH. Early treatment of vertical skeletal dysplasia: the hyperdivergent phenotype. Am J Orthod Dentofacial Orthop. 2000;118:317-27.

15. English JD. Early treatment of skeletal open bite malocclusions. Am J Orthod Dentofacial Orthop. 2002;121:563-5. 
16. Işcan HN, Dinçer M, Gültan A, Meral O, Taner-Sarisoy L. Effects of vertical chincap therapy on the mandibular morphology in open-bite patients. Am J Orthod Dentofacial Orthop. 2002;122:506-11.

17. Kiliaridis S, Egermark I, Thilander B. Anterior open bite treatment with magnets. Eur J Orthod. 1990;12:447-57.

18. Kuster R, Ingervall B. The effect treatment of skeletal open bite with two types of bite block. Eur J Orthod. 1992;14:489-99.

19. Isscan HN, Akkaya S, Elcin K. The effect of spring-loaded posterior bite block on the maxillo-facial morphology. Eur J Orthod. 1992;14:54-60.

20. Işcan HN, Sarisoy L. Comparison of the effects of passive posterior biteblocks with different construction bites on the craniofacial and dentoalveolar structures. Am J Orthod Dentofacial Orthop. 1997;112:171-8.

21. Bazzucchi A, Hans MG, Nelson S, Powers M, Parker S. Evidence of correction of open bite malocclusion using active vertical corrector treatment. Semin Orthod. 1999:5:110-20.

22. Cinsar A, Alagha AR, Akyalcin S. Skeletal open bite correction with rapid molar intruder appliance in growing individuals. Angle Orthod. 2007;77:632-9.

23. Doshi UH, Bhad WA. Spring-loaded bite-blocks for early correction of skeletal open bite associated with thumb sucking. Am J Orthod Dentofacial Orthop. 2011:140:115-20.

24. Albogha MH, Takahashi I, Sawan MN. Early treatment of anterior open bite: comparison of the vertical and horizontal morphological changes induced by magnetic bite-blocks and adjusted rapid molar intruders. Korean J Orthod. 2015:45:38-46.

25. Ingervall B, Bitsanis E. A pilot study of the effect of masticatory muscle training on facial growth in long-face children. Eur J Orthod. 1987:9:15-23.

26. Fränkel $\mathrm{R}$, Fränkel $\mathrm{C}$. Functional aspects of molar extraction in skeletal open bite. In: Graber LW, Graber TM, editors. Orthodontics: State of the Art: Essence of the Science. Mosby: St Louis; 1986. p. 184-99.

27. Pedrin F, Almeida MR, Almeida RR, Almeida-Pedrin RR, Torres F. A prospective study of the treatment effects of a removable appliance with palatal crib combined with high-pull chincup therapy in anterior open-bite patients. Am J Orthod Dentofacial Orthop. 2006;129:418-23.

28. Torres F, Almeida RR, de Almeida MR, Almeida-Pedrin RR, Pedrin F, Henriques JF. Anterior open bite treated with a palatal crib and high-pull chin cup therapy. A prospective randomized study. Eur J Orthod. 2006;28:610-7.

29. Cozza P, Baccetti T, Franchi L, McNamara Jr JA. Treatment effects of a modified quad-helix in patients with dentoskeletal open bites. Am J Orthod Dentofacial Orthop. 2006;129:734-9.

30. Cozza P, Mucedero M, Baccetti T, Franchi L. Treatment and posttreatment effects of quad-helix/crib therapy of dentoskeletal open bite. Angle Orthod. 2007;77:640-5.

31. Cozza P, Baccetti T, Franchi L, Mucedero M. Comparison of 2 early treatment protocols for open-bite malocclusions. Am J Orthod Dentofacial Orthop. 2007:132:743-7.

32. Giuntini V, Franchi L, Baccetti T, Mucedero M, Cozza P. Dentoskeletal changes associated with fixed and removable appliances with a crib in open-bite patients in the mixed dentition. Am J Orthod Dentofacial Orthop. 2008;133:77-80.

33. Torres FC, Almeida RR, Almeida-Pedrin RR, Pedrin F, Paranhos LR. Dentoalveolar comparative study between removable and fixed cribs, associated to chincup, in anterior open bite treatment. J Appl Oral Sci. 2012; 20:531-7.

34. Cassis MA, de Almeida RR, Janson G, de Almeida-Pedrin RR, de Almeida MR. Treatment effects of bonded spurs associated with high-pull chincup therapy in the treatment of patients with anterior open bite. Am J Orthod Dentofacial Orthop. 2012;142:487-93.

35. Mucedero M, Franchi L, Giuntini V, Vangelisti A, Mc Namara Jr JA, Cozza P. Stability of quad-helix/crib therapy in dentoskeletal open bite: a long-term controlled study. Am J Orthod Dentofacial Orthop. 2013;143:695-703.

36. Fränkel $R$, Fränkel $C$. A functional approach to treatment of skeletal open bite. Am J Orthod. 1983:84:54-68.

37. Haydar B, Enacar A. Functional regulator therapy in treatment of skeletal open-bite. J Nihon Univ Sch Dent. 1992;34:278-87.

38. Ngan P, Wilson S, Florman M, Wei S. Treatment of class II open bite in the mixed dentition with a removable functional appliance headgear. Quintessence Int. 1992;23:323-33.

39. Weinbach JR, Smith RJ. Cephalometric changes during treatment with the open bite Bionator. Am J Orthod Dentofacial Orthop. 1992;101:367-74.
40. Erbay $E$, Ugur T, Ulgen M. The effects of Fränkel's function regulator (FR-4) therapy on the treatment of angle class I skeletal anterior open bite malocclusion. Am J Orthod Dentofacial Orthop. 1995;108:9-21.

41. Defraia E, Marinelli A, Baroni G, Franchi L, Baccetti T. Early orthodontic treatment of skeletal open-bite malocclusion with the open-bite bionator: a cephalometric study. Am J Orthod Dentofacial Orthop. 2007;132:595-8.

42. Feres MF, Abreu LG, Insabralde NM, Almeida MR, Flores-Mir C. Effectiveness of the open bitetreatment in growing children and adolescents. A systematic review. Eur J Orthod. 2015. [Epub ahead of print].

43. Lentini-Oliveira DA, Carvalho FR, Rodrigues CG, Ye Q, Hu R, Minami-Sugaya $\mathrm{H}$, Carvalho LBF, et al. Orthodontic and orthopaedic treatment for anterior open bite in children. Cochrane Database Syst Rev. 2014;9:CD005515.

44. Moher D, Liberati A, Tetzlaff J, Altman DG, The PRISMA Group. Preferred Reporting Items for Systematic Reviews and Meta-Analyses: the PRISMA statement. PLoS Med. 2009;6(6):e1000097.

45. National Health Service (NHS) Centre for Reviews and Dissemination Report number 4. Undertaking systematic reviews of research on effectiveness. 2nd ed. University of York: York Publishing Services; 2001. Available at: http:// www.york.ac.uk/inst/crd/crdrep.htm. 2004.

46. Antczak AA, Tang J, Chalmers TC. Quality assessment of randomized control trials in dental research I. Methods. J Periodontal Res. 1986;21:305-14.

47. Jadad AR, Moore RA, Carroll D, Jenkinson C, Reynolds DJ, Gavaghan DJ, McQuay HJ. Assessing the quality of reports of randomized clinical trials: is blinding necessary? Control Clin Trials. 1996;17:1-12.

48. Petrén S, Bondemark L, Söderfeldt BB. A systematic review concerning early orthodontic treatment of unilateral posterior crossbite. Angle Orthod. 2003; 73:588-96.

49. Kiriakou J, Pandis N, Madianos P, Polychronopoulou A. Developing evidence-based dentistry skills: how to interpret randomized clinical trials and systematic reviews. Prog Orthod. 2014;15:58.

50. Riolo ML, Moyers RE, McNamara Jr JA, Hunter WS. An atlas of craniofacial growth: cephalometric standards from The University School Growth Study, The University of Michigan. craniofacial growth series, vol. 2. Ann Arbor: Center for Human Growth and Development, The University of Michigan; 1974

\section{Submit your manuscript to a SpringerOpen ${ }^{\circ}$ journal and benefit from:}

- Convenient online submission

- Rigorous peer review

- Immediate publication on acceptance

- Open access: articles freely available online

- High visibility within the field

Retaining the copyright to your article

Submit your next manuscript at $>$ springeropen.com 